\title{
Neuropatía por atrapamiento del nervio supraescapular: reporte de dos casos.
}

\author{
Supracapular nerve entrapment neuropathy: report of two cases. \\ Neuropatía of atrapamiento del nervio supraescapular: reporte de dos \\ casos.
}

Fernando Martínez Neurocirujano. Prof. Agregado Neurocirugía. UdelaR

Resumen: La neuropatía por atrapamiento del nervio supraescapular (NSE) es relativamente poco frecuente, sin embargo, debe ser identificada ya que es una causa potencialmente tratable de hombro doloroso. Se reportan dos casos clínicos tratados mediante cirugía. Se trata de dos hombres de 42 y 56 años que consultaron por dolor y limitación funcional para realizar movimientos de abducción y rotación externa del hombro derecho. El caso 2 presentaba un claro déficit motor (M2 de la escala del British Medical Council). Debido a la ausencia de mejoría con rehabilitación, fue necesario realizar descompresión quirúrgica medianteabordaje posterior con resultado satisfactorio en ambos casos. En la presente revisión, se destaca la importancia del diagnóstico y tratamiento precoces de esta neuropatía compresiva, ya que su progresión puede resultar en atrofia muscular, con la consecuente limitación funcional del hombro por recuperación incompleta a pesar de un tratamiento adecuado.

Palabras clave: Nervio supraescapular, Neuropatía por atrapamiento, Hombro doloroso.

Abstract: Neuropathy due to entrapment of the suprascapular nerve (SSN) is relatively rare, however, it should be known because it is a potentially treatable cause of painful shoulder. In this report two clinical cases treated by surgery are presented. These were two men aged 42 and 56 who consulted for pain and functional impotence for abduction and external rotation of the shoulder. Case 2 had a clear motor deficit (M2 of the BMC scale). Since they did not improve with physical rehabilitation, posterior decompression was performed with satisfactory results in both cases. Early diagnosis is of paramount importance to avoid its progression, since if there is muscle atrophy there may be incomplete recovery of abduction and external rotation of the shoulder.

Keywords: Suprascapularnerve, Entrapmentneuropathy, Painfullshoulder.

Resumo: A neuropatia por atrapalhamento do nervo supraescapular (NSE) é um dos aspectos mais importantes do enfoque, mas é identificada como uma das causas mais complexas do hombro doloroso. Se reportan em casos omissos, por exemplo, cirugía. Se trata de hombres de 42 e 56 anos de consulta sobre a dolorosidade e a limitação funcional para realizar movimentos de rapto e rotação externa do homo derecho. O caso 2 apresenta um claro déficit motor (M2 da escala do British Medical Council). Debido a ausencia de mejoría con rehabilitación, fue necesario realizar descompresión quiroptería mediante abordar posterior con satisfatorio de resultado en ambos os casos. No presente relatório, é importante a importância do diagnóstico e tratamento precoces desta neuropatia periférica, o que resulta na sua progressão e atrofia muscular, com a consequente limitação funcional do processo de recuperação incompleta de um tratamento adecuado.

Palavras-chave: Nervio supraescapular, Neuropatía por atrapamiento, Hombro doloroso. 
Recibido: 13/03/2018 - Aceptado: 01/06/2018

Servicio de Neurocirugía. Hospital de Clínicas. Facultad de Medicina. Universidad de la República. Servicio de

Neurocirugía. Asociación Española Primera de Socorros Mutuos. Institución Asistencia Médica Colectiva. Montevideo

Uruguay

Correspondencia: E-mail:fermartneuro@gmail.com 


\section{Introducción}

La neuropatía compresiva del nervio supraescapular (NSE) es una entidad poco frecuente. Su diagnóstico se basa en un correcto interrogatorio y examen clínico, pero dada su baja frecuencia, no es sospechada habitualmente.

Sin embargo, representa el $1 \%$ de todas las causas de dolor en la región del hombro ${ }^{(1)}$. Este síntoma es un motivo de consulta relativamente frecuente en traumatología.

En este artículo se reportan dos casos de neuropatía del NSE tratados quirúrgicamente y se realiza una revisión del tema.

\section{Casos clínicos}

\section{a) Caso clínico 1}

Hombre de 42 años que consultó por presentar dolor en región del hombro derecho de 3 meses de evolución, agravado en los movimientos de rotación externa y abducción del hombro, que le impedía hacer deportes (natación). Los movimientos de rotación y abducción del hombro le generaban un aumento del dolor. El examen clínico evidenció hipoestesia en la región deltoidea y dolor durante la abducción del hombro, con un déficit motor leve (M4 del British Medical Council [BMC]).

Se le realizó el Score DASH (Disabilities of Arm, Shoulder and Hand) sumando 32.5 puntos. El estudio eléctrico (EE) mostró una denervación activa sin signos de reinervación en músculos supra e infraespinosos por lo que se estableció el diagnóstico de atrapamiento del NSE.

Debido a la presencia de debilidad muscular y ausencia de mejoría a pesar de la rehabilitación, se realizó liberación del nervio por abordaje posterior en posición de decúbito lateral (Figura 1).

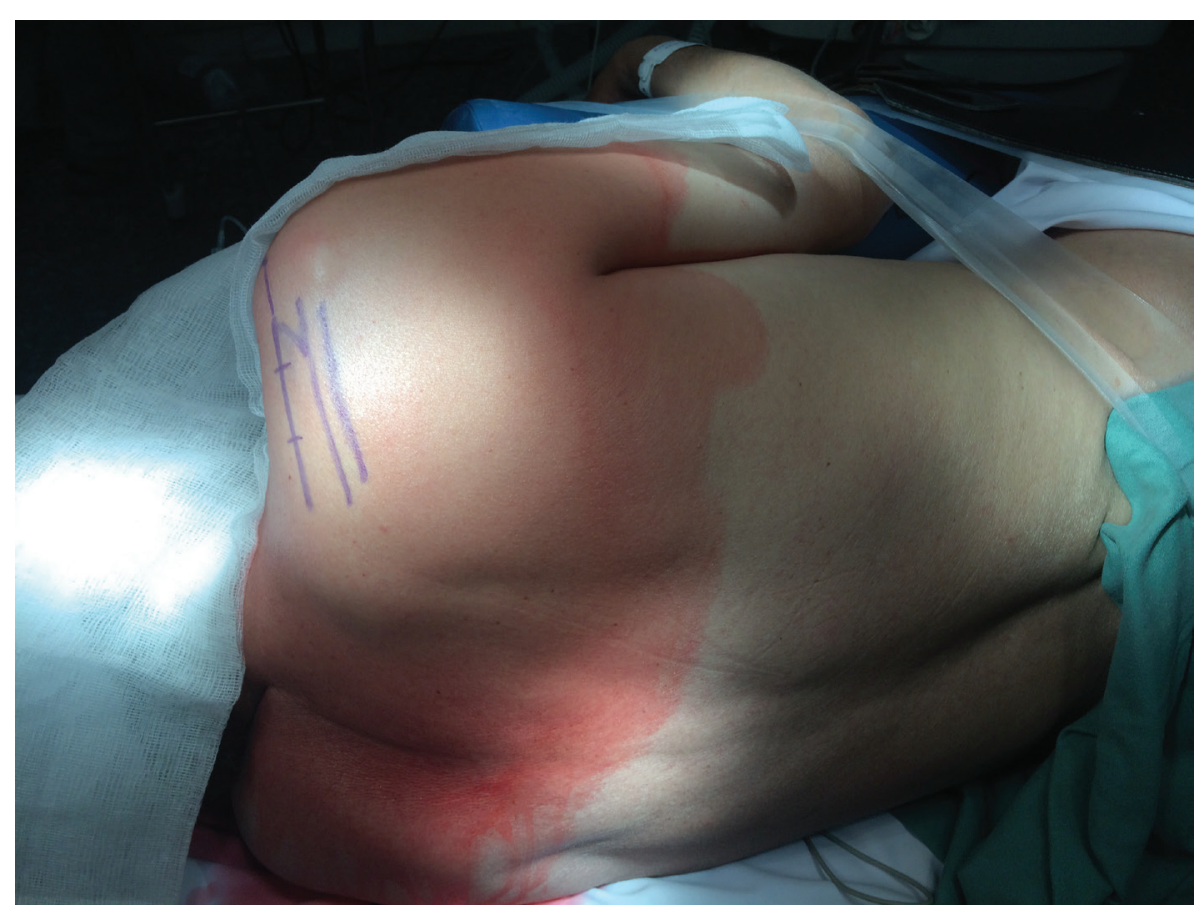

El paciente refirió mejoría en el período postoperatorio inmediato en lo referido a dolor. Transcurridos 6 meses, el puntaje DASH fue 0 y la recuperación de la fuerza muscular fue completa.

\section{b) Caso clínico 2}

Hombre de 56 años con historia de 6 meses de evolución de intenso dolor en hombro e impotencia funcional para la abducción y rotación externa a derecha (M2). El puntaje en el Score DASH fue de 70. El EE mostró denervación crónica sin signos de reinervación en músculos supraespinoso e infraespinoso. Se realizó una Imagen por Resonancia Magnética 
(IRM) de hombro que evidenció cambiosen la intensidad de señal en ambos músculos y atrofia en músculos supraespinoso e infraespinoso (figura 2). Una vez confirmado el diagnóstico de neuropatía del NSE se realizó descompresión quirúrgica por abordaje posterior presentando ulteriormente mejoría del dolor. Luego de intensa rehabilitación, en seis meses la motilidad activa era prácticamente normal, y su puntaje DASH fue de 10.

Figura 2: IRM de hombro derecho

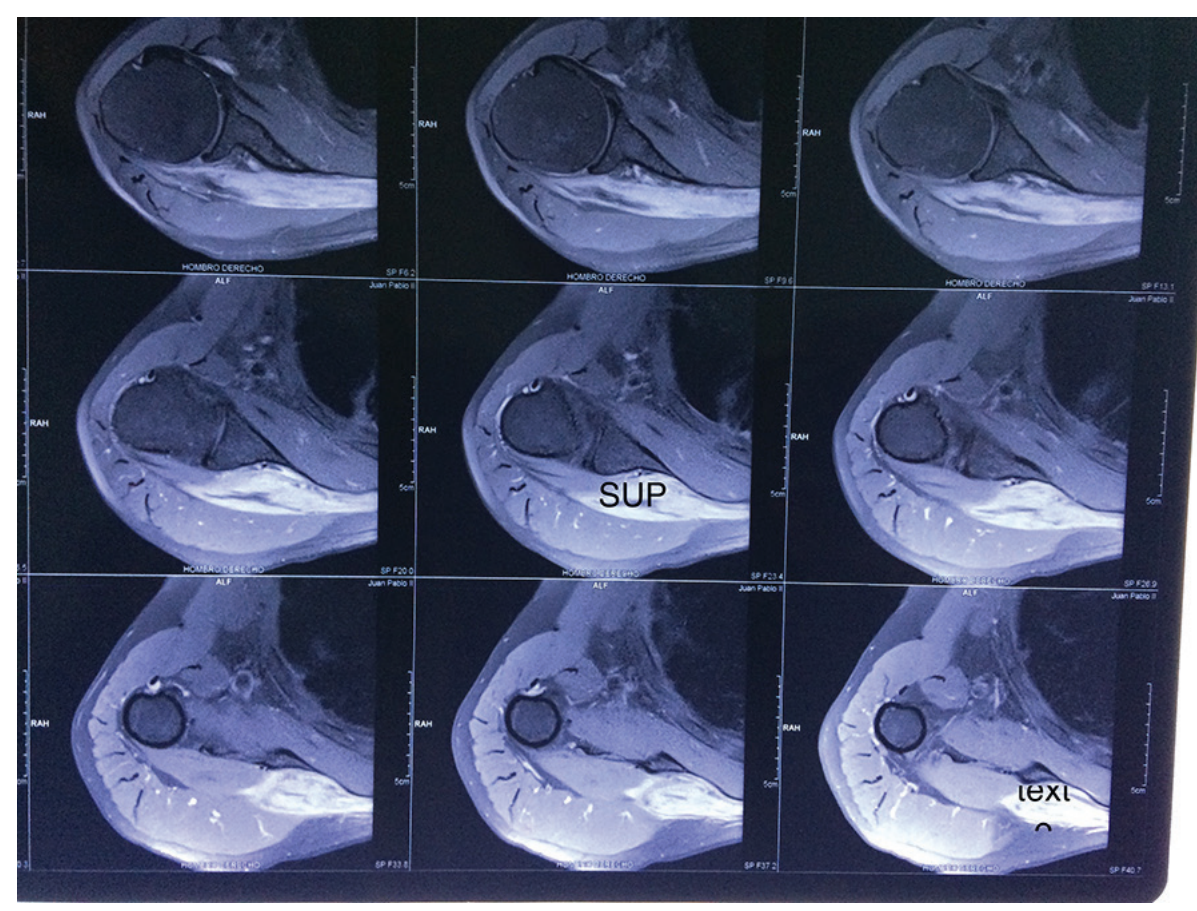

\section{Discusión}

La neuropatía del NSE fue descrita por André Thomas en 1936 y es una de las múltiples causas de dolor en la región del hombro ${ }^{(1-4)}$.

Es una neuropatía por atrapamiento poco conocida y en general, de baja incidencia. Por ejemplo, Zehetgruber y cols ${ }^{(3)}$ en un meta-análisis publicado en 2002 , encontraron 88 casos reportados entre 1959 y 2001.

Desde el punto de vista clínico, generalmente, luego de una etapa de dolor los pacientes con neuropatía del NSE refieren parestesias y/o hipoestesia en la región del hombro y compromiso motor en los músculos supra e infraespinoso. Esto produce una limitación en la abducción y rotación externa del hombro y es una de las claves diagnósticas. Puede existir también dolor difuso en el hombro por sobrecarga muscular o compromiso de la rama articular del NSE ${ }^{(5)}$. Sin embargo, otras entidades patológicas pueden dar cuadros parecidos, como: la artrosis de hombro, el sufrimiento o rotura del manguito rotador, radiculopatías de C5, artritis acromioclavicular, síndrome de Parsonage-Turner, Sindrome de Pancoast, tumores de partes blandas de la región ${ }^{(3-8)}$. Es por eso que se debe tener en mente la neuropatía del NSE para poder diagnosticarla.

El compromiso del NSE se debe en general a la compresión del mismo en su pasaje por la escotadura escapular o a nivel del ligamento espinoglenoideo. Esta última eventualidad es menos frecuente y produce solo compromiso del músculo infraespinoso. El diagnóstico diferencial entre estos dos puntos de compresión se basa en la clínica y la neurofisiología ${ }^{(2,6)}$.

Entre los factores de riesgo descritos para padecer una neuropatía por atrapamiento del NSE se citan: la morfología de la escotadura escapular, la osificación del ligamento transverso de la escápula, deportes que exijan elevar de forma repetida al brazo por arriba de la cabeza, traumatismos, fracturas de clavícula o escápula, procedimientos quirúrgicos en la región y gangliones articulares, entre otras causas ${ }^{(1,4,9)}$.

En nuestros casos identificamos como factor de riesgo el deporte en uno de los pacientes (natación y remo) y en el otro, las tareas de esfuerzo repetitivas (trabajador rural). 
El correcto interrogatorio y examen físico deben sugerir la sospecha de neuropatía del NSE y dicha presentación clínica típica es el dolor en la región deltoidea, trastornos sensitivos en dicha área y déficit funcional en los músculos supra e infraespinoso. El dolor puede abarcar áreas más amplias por sobrecarga de otros grupos musculares, dado que supra e infraespinoso colaboran de forma activa en la rotación externa y abducción del hombro, así como en la estabilidad de la articulación escápulohumeral.

Dentro de la paraclínica, el EE es fundamental ya que permite evidenciar el decremento en la velocidad de conducción del nervio y en electromiograma mostrar signos precoces de denervación (que preceden a la atrofia muscular en supra e infraespinoso). Igualmente, la neurofisiología puede ser muy útil en identificar el sitio de atrapamiento del nervio en las dos topografías posibles: ligamento transverso o ligamento espinoglenoideo. En la primer sitio de compresión (proximal), el compromiso se verá en los músculos supra e infraespinoso en tanto que en la compresione a nivel del ligamento espinoglenoideo (distal) se verá afectado únicamente el músculoninfraespinoso ${ }^{(2)}$. En este grupo de pacientes es más frecuente encontrar gangliones como causa de compresión, por lo que se justificaría claramente hacer estudios de imagen ${ }^{(3)}$.

Los estudios de imagen son útiles para descartar los diagnósticos diferenciales y eventualmente, confirmar el de neuropatía del NSE, como ocurrió en nuestro segundo caso. En este paciente se hizo una IRM de hombro debido a la sospecha de lesión del manguito rotador, sin embargo el estudio evidenció cambios en la intensidad de señal por denervación y atrofia en los músculos supra e infraespinoso. Este hallazgo es típico de la neuropatía del NSE en IRM (10). A su vez, este estudio permite descartar causas secundarias de compresión ${ }^{(10,11)}$.

La ecografía puede resultar muy útil si se realiza por un técnico entrenado, ya que puede mostrar el calibre del nervio (engrosado por trauma repetitivo), la presencia de gangliones $u$ otros tumores de la región, osificación del ligamento transverso de la escápula y eventualmente, cambios en la ecogenicidad muscular secundarios a atrofia o infiltración grasa ${ }^{(12)}$.

Como en toda neuropatía por atrapamiento en la que no hay atrofia muscular o debilidad importante, se debe optar primero por el tratamiento fisiátrico y si este falla, la opción es la cirugía.

En ambos se optó por el tratamiento quirúrgico dado que presentaban signos motores, uno de ellos con una atrofia y disminución de la fuerza muscular.

La cirugía es relativamente sencilla y consiste en la liberación del nervio en su trayecto por debajo del ligamento transverso de la escápula. Dadas las variantes anatómicas de la escotadura escapular, se debe tener en cuenta que el ligamento puede estar calcificado o la escotadura puede ser de tamaño reducido. Igualmente, existen varias publicaciones que describen la anatomía quirúrgica y puntos de referencia anatómicos para localizar la escotadura escapular de forma sencilla ${ }^{(13-15)}$.

En condiciones normales el nervio pasa por debajo del ligamento junto con las venas en tanto la arteria pasa por arriba del ligamento. Luego de cortar el mismo se verifica que el nervio quede libre y se puede inspeccionar la región en busca de neuromas o gangliones que lo compriman.

Se describen en la literatura algunos casos de decompresión por vía endoscópica ${ }^{(11)}$. No hay suficiente evidencia científica para poder comparar la vía abierta con la vía endoscópica, sin embargo los resultados estéticos de la vía clásica son muy aceptables.

\section{Conclusiones}

Se presentan dos casos de neuropatía del nervio supraescapular. La misma es una causa poco frecuente de hombro doloroso, sin embargo debe ser conocida porque las demoras terapéuticas pueden producir una recuperación incompleta.

Los pilares diagnósticos son el examen clínico, el estudio eléctrico y la imagenología (IRM o ecografía) 


\section{Bibliografía}

1- Labętowicz P, Synder M, Wojciechowski M, Orczyk K, Jezierski H, Topol M, et al. Protective and Predisposing Morphological Factors in Suprascapular Nerve Entrapment Syndrome: A Fundamental Review Based on Recent Observations. Biomed Res Int. 2017;2017:4659761. doi: $10.1155 / 2017 / 4659761$.

2- Padua L, Conti V, Pazzaglia C, Caliandro P. About neurophysiological evaluation in suprascapular nerve entrapment and methods of review. Int Orthop. 2003;27(3):194.

3- Zehetgruber H, Noske H, Lang T, Wurnig C. Suprascapular nerve entrapment. A meta-analysis. Int Orthop. 2002;26(6):339-43.

4- Witvrouw E, Cools A, Lysens R, Cambier D, Vanderstraeten G, Victor J, et al. Suprascapular neuropathy in volleyball players. Br J Sports Med. 2000;34(3):174-80.

5- Liveson JA, Bronson MJ, Pollack MA. Suprascapular nerve lesions at the spinoglenoid notch: report of three cases and review of the literature. J Neurol Neurosurg Psychiatry. 1991;54(3):241-3.

6- Rizzello G, Longo UG, Trovato U, Fumo C, Khan WS, Maffulli N, et al. Bilateral suprascapular nerve entrapment by ganglion cyst associated with superior labral lesion. Open Orthop J. 2013;7:129-32.

7- Dikici AS, Bakan S, Kandemirli SG, Sonmez S, Ersen E, Comunoglu N, et al. Suprascapular nerve compression due to rib osteochondroma: MR imaging features. Diagn Interv Imaging. 2016;97(1):10911. doi: 10.1016/j.diii.2015.05.007.

8- Ishimaru D, Nagano A, Terabayashi N, Nishimoto Y, Akiyama H. Suprascapular Nerve Entrapment Caused by Protrusion of an Intraosseous Ganglion of the Glenoid into the Spinoglenoid Notch: A Rare Cause of Posterior Shoulder Pain. Case Rep Orthop. 2017;2017:1704697. doi: 10.1155/2017/1704697.

9- Neal S, Fields KB. Peripheral nerve entrapment and injury in the upper extremity. Am Fam Physician. 2010;81(2):147-55.

10- Dong Q, Jacobson JA, Jamadar DA, Gandikota G, Brandon C, Morag Y, et al. Entrapment neuropathies in theupper and lowerlimbs: anatomy and MRI features. Radiol Res Pract. 2012; 230679. doi: $10.1155 / 2012 / 230679$.

11- Bruce J, Dorizas J. Suprascapular nerve entrapment due to a stenotic foramen: a variant of the suprascapular notch. Sports Health. 2013;5(4):363-6.

12- Kowalska B, Sudoł-Szopińska I. Ultrasound assessment on selected peripheral nerve pathologies. Part I: Entrapment neuropathies of the upper limb - excluding carpal tunnel syndrome. J Ultrason. 2012;12(50):307-18. doi: 10.15557/JoU.2012.0016.

13- Martínez F, Jaume A, Sienra C, Ruso A. Anatomía quirúrgica de la transferencia nerviosa de espinal accesorio a nervio supraescapular por vía posterior. Rev. latinoam. Neurocir. 2017;26(1): 6.

14- Martínez F. Technical Note: Spinal-Accessory to Suprascapular Nerve Transfer by Posterior Approach. Austin Neurosurg Open Access. 2017; 4(1): 1058.

15- Colbert SH, Mackinnon S. Posterior approach for double nerve transfer for restoration of shoulder function in upper brachial plexus palsy. Hand. 2006;1(2):71-77. 